\title{
The screw dynamo in a time-dependent pipe flow
}

\author{
Wolfgang Dobler \\ Kiepenheuer-Institut für Sonnenphysik, Schöneckstraße 6, D-79104 Freiburg, GERMANY* \\ Peter Frick and Rodion Stepanov \\ Institute of Continuous Media Mechanics, 1, Korolev, Perm, 614061, RUSSIA
}

(Dated: December 13, 2018)

\begin{abstract}
The kinematic dynamo problem is investigated for the flow of a conducting fluid in a cylindrical, periodic tube with conducting walls. The methods used are an eigenvalue analysis of the steady regime, and the three-dimensional solution of the time-dependent induction equation. The configuration and parameters considered here are close to those of a dynamo experiment planned in Perm, which will use a torus-shaped channel. We find growth of an initial magnetic field by more than 3 orders of magnitude. Marked field growth can be obtained if the braking time is less than $0.2 \mathrm{~s}$ and only one diverter is used in the channel. The structure of the seed field has a strong impact on the field amplification factor. The generation properties can be improved by adding ferromagnetic particles to the fluid in order to increase its relative permeability, but this will not be necessary for the success of the dynamo experiment. For higher magnetic Reynolds numbers, the nontrivial evolution of different magnetic modes limits the value of simple 'optimistic' and 'pessimistic' estimates.
\end{abstract}

PACS numbers: 47.65.+a, 07.55.Db, 61.25.Mv

\section{INTRODUCTION}

The screw dynamo is a simple dynamo model that has been extensively studied in dynamo theory. It is based on an idea due to Lortz 1] and Ponomarenko 2], according to which magnetic field can be generated by the helical motion of a rigid, electrically conducting cylinder of infinite length through an infinitely extended medium of equal conductivity. This problem implies a simple velocity field and leads to a critical magnetic Reynolds number as low as 3

$$
\mathrm{Rm}=\frac{U r_{0}}{\eta}=17.7
$$

where $U$ is the (constant) longitudinal velocity of the cylinder, $r_{0}$ is its radius and $\eta$ is the magnetic diffusivity. This dynamo model has a discontinuous velocity profile, and will be referred to as the 'Ponomarenko dynamo'. More realistic models of the screw dynamo, involving continuous and hydrodynamically realistic velocity fields were considered by several authors. References [4, 5] develop a very accurate asymptotic theory for the screw dynamo in smooth flows, which has been complemented by numerical simulations [6]. In [7], this theory is applied to a number of realistic flows. Reference 8 extended the numerical analysis to flows fluctuating in time. Other time dependent screw dynamo models were presented in [9] in connection with the Perm dynamo experiment. Reference [10] has put the screw dynamo into a larger context of slow dynamo mechanisms and 11] have generalized the concept to non-axisymmetric flows. The

*Electronic address: Wolfgang.Dobler@kis.uni-freiburg.de

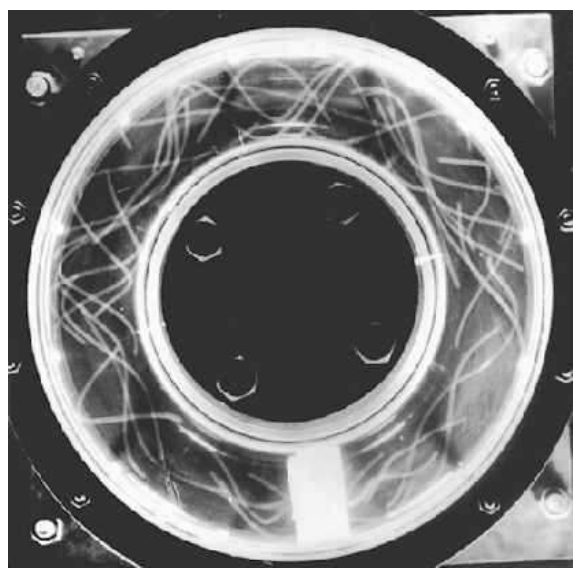

FIG. 1: Photograph of a water experiment showing streamlines in an initially spinning torus after abrupt braking. The white object in the channel is the diverter, a kind of a fixed ship screw that makes the motion strongly helical. From Ref. [9].

nonlinear behavior of the screw dynamo for spiral Couette flow has been investigated with asymptotic methods by 12$]$ and numerically by 13$]$.

We are interested in the screw dynamo problem in the context of a new experimental dynamo project 9 ]. The basic idea of this project, introduced in 14], is to realize the dynamo effect in a strongly time-dependent helical flow. The flow is generated in a quickly rotating toroidal channel after abrupt braking, and is shown, for a water experiment, in Fig. 1] This application raises new questions concerning the screw dynamo, which have not been addressed in previous studies. In particular, the flow of the conducting fluid will be located in a closed channel and will be supercritical during a short time interval only. This requires the solution of the induction equation 
for three-dimensional geometry in a time-dependent flow. Moreover, in oder to understand the saturation of the magnetic field (if it occurs during the experiment), one has to investigate the time evolution of the full nonlinear MHD equations. The experimental scheme also requires strong optimization of the channel (minimization of its mass under optimal conductivity and wall thickness).

In this paper we investigate the screw dynamo in a time-dependent flow using two different methods which allow different questions to be addressed. First, the analysis of the eigenvalue problem related to the case of a steady velocity field gives insight into the full spectral structure and is numerically the most efficient approach. Our second method - numerical solution of the threedimensional, space- and time-dependent problem - is numerically much more demanding, but it allows us to investigate the full three-dimensional structure and is the only one that has the perspective of tackling the nonlinear problem. While the final goal is to solve the fully nonlinear problem and to include all geometrical and dynamical effects, the present paper only discusses the linear phase of magnetic field growth in (periodic) cylindrical geometry.

Most laboratory dynamo projects use liquid sodium as conducting fluid, which has a magnetic Prandtl number $\mathrm{Pm}=\nu / \eta \approx 10^{-5}$. This means that, in order to achieve the critical magnetic Reynolds number of a few tens, one operates at kinematic Reynolds number of order $10^{6}-10^{7}$, which is far beyond what can be numerically simulated even on the largest computers. Thus, we cannot solve the dynamical part of the problem consistently and in the current paper just use mean velocity profiles as inferred from experiments.

The structure of the paper is as follows. Section III specifies the equations and geometry of the problem addressed here. In Sec. IIII we present numerical solutions of the induction equation for prescribed, steady velocity profiles similar to those found in cylindrical channels. If the velocity field is axisymmetric and identical in any cross section through the cylinder/pipe, the problem can be reduced to a one-dimensional eigenvalue problem that is solved numerically by discretization. This approach is sufficiently efficient to allow us to scan the space of relevant parameters and isolate the cases that will be most favorable for the realization of the experiment. Section IV presents results obtained with a three-dimensional MHD code solving the induction problem for a velocity field that varies in space and time. We finally draw some conclusions about the realizability of the planned dynamo experiment in Sec. D

\section{FORMULATION OF THE PROBLEM}

The proposed Perm dynamo experiment [14] will implement a helical, strongly time-dependent flow of liquid sodium through a torus, surrounded by a thin shell of copper, the electrical conductivity of which is about five times higher than that of liquid sodium. The role of this conducting shell is to 'anchor' the magnetic field lines in the exterior frame, so that the shear due to the velocity difference between flow and exterior can enhance the magnetic field.

Mathematically, dynamo action is characterized by the presence of growing solutions $\mathbf{B}(\mathbf{x}, t)$ of the induction equation

$$
\frac{\partial \mathbf{B}}{\partial t}=\nabla \times\left[\left(\mathbf{v}+\eta \nabla \ln \mu_{\mathrm{r}}\right) \times \mathbf{B}-\eta \nabla \times \mathbf{B}\right],
$$

satisfying the solenoidality condition

$$
\nabla \cdot \mathbf{B}=0 .
$$

Here $\mathbf{B}$ denotes the magnetic flux density, $\mathbf{v}$ the velocity field, $\mu_{0}$ and $\mu_{\mathrm{r}}$ are the magnetic vacuum permeability and the relative magnetic permeability of the medium, and $\eta$ is the magnetic diffusivity of the fluid, related to the electrical conductivity $\sigma$ by $\eta=\left(\mu_{0} \mu_{\mathrm{r}} \sigma\right)^{-1}$. The term $\eta \nabla \ln \mu_{\mathrm{r}} \equiv \mathbf{V}_{\mathrm{p}}$ arises if the relative magnetic permeability is a function of position and gives rise to what we call "paramagnetic pumping" (with an effective velocity $\mathbf{V}_{\mathrm{p}}$ ) of magnetic flux into the regions of enhanced permeability. A self-consistent description would also include the Navier-Stokes equation

$$
\frac{\partial \mathbf{v}}{\partial t}=-(\mathbf{v} \cdot \nabla) \mathbf{v}-\frac{\nabla p}{\varrho}+\frac{\mathbf{j} \times \mathbf{B}}{\varrho}+\nu \Delta \mathbf{v},
$$

together with the condition

$$
\nabla \cdot \mathbf{v}=0
$$

for an incompressible fluid. Here $p$ denotes pressure, $\varrho$ density, $\mathbf{j} \equiv \nabla \times\left(\mathbf{B} / \mu_{0} \mu_{\mathrm{r}}\right)$ the electric current density and $\nu$ is the kinematic viscosity. The equations given above are complemented by boundary conditions describing the properties of the walls (see 14]).

In the present paper, we restrict ourselves to the kinematic dynamo problem, i.e., we consider only Eqs. (2) and (3), using a given solenoidal velocity field $\mathbf{v}(\mathbf{x}, t)$ and neglect the magnetic back-reaction through the Lorentz force. This leads to a linear problem in $\mathbf{B}$ and is very helpful to understand the evolution for weak and moderately strong magnetic fields. This approach will allow for optimization of the experimental apparatus in many respects since the kinematic growth of $\mathbf{B}$ over several orders of magnitude is necessary for the success of the experiment.

The turbulent flow of a conducting medium will give rise to turbulent induction effects that can be estimated by mean-field theory. In the present paper, we neglect these extra terms in the induction equation (2) and refer the reader to the discussion in Ref. [15].

While the curvature of the pipe can be expected to play a role for the geometry of the experiment (the ratio of outer radius $R$ to inner radius $r_{0}$ of the torus being about 


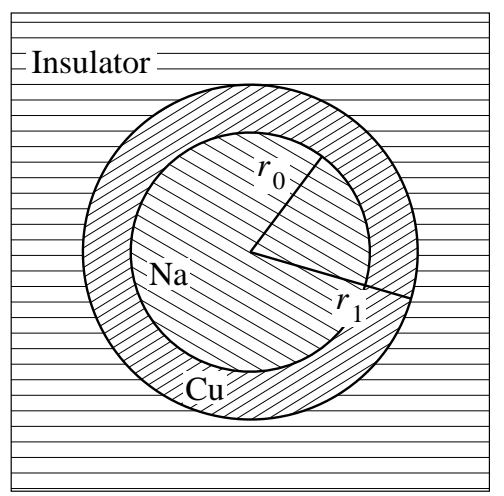

FIG. 2: Cross section of the torus/pipe. The interior of the pipe, $r<r_{0}$, is filled with a liquid (sodium) of electrical conductivity $\sigma_{\mathrm{f}}$, the solid shell $r_{0}<r<r_{1}$ (made of copper) has a conductivity $\sigma_{\mathrm{sh}}$ and is itself surrounded by an electrically insulating medium.

3), we currently neglect it by considering a cylindrical pipe instead of a torus. Connecting the two ends of the cylinder by periodic boundary conditions and setting its length equal to $L_{z}=2 \pi R$, we obtain a reasonable first approximation to torus geometry.

\section{ONE-DIMENSIONAL, TIME-INDEPENDENT PROBLEM}

\section{A. Equations}

Let us consider the evolution of the magnetic field in a given helical flow through a cylindrical, conducting tube with finite wall thickness $d=r_{1}-r_{0}$ (the inner radius of the tube is $r_{0}$, the outer $r_{1}$ ), surrounded by an electrical insulator; Fig. 2 shows a cross section through the pipe. We adopt cylindrical coordinates $(r, \varphi, z)$ and assume an axisymmetric helical velocity field $\mathbf{v}(r)=\left[0, r \omega(r), v_{z}(r)\right]$. The magnetic diffusivity and permeability may vary as functions of radius, $\eta=\eta(r)$, $\mu_{\mathrm{r}}=\mu_{\mathrm{r}}(r)$.

Solutions of the kinematic dynamo problem can be represented as a superposition of individual modes with exponential growth. Because the coefficients in the equations depend on $r$ only, we are looking for solutions in the form of a propagating helical wave

$$
\mathbf{B}(r, \varphi, z, t)=\mathbf{b}(r) e^{\gamma t+i(m \varphi+k z)}
$$

where $\gamma$ appears as an eigenvalue and is in general a complex quantity. The real part of $\gamma$ determines whether B decays $(\Re \gamma<0)$ or grows $(\Re \gamma>0)$. For a given mode, there exists a critical value $\mathrm{Rm}_{*}$ of the magnetic Reynolds number (11) for which $\Re \gamma$ changes sign from negative to positive. The lowest value of $\mathrm{Rm}_{*}$ is the threshold for dynamo action. For the type of dynamo problem considered here, it is typically between 10 and 100 (see Fig. 5 below for an illustration).
Inserting Eq. (6) into the induction equation (2), one gets

$$
\begin{gathered}
\gamma b_{r}+i\left(m \omega+k v_{z}\right) b_{r}+V_{\mathrm{p}} \frac{d\left(r b_{r}\right)}{r d r} \\
=\mathrm{Rm}^{-1}\left[\eta\left(\hat{D} b_{r}-\frac{2 i m}{r^{2}} b_{\varphi}\right)\right] \\
\gamma b_{\varphi}+i\left(m \omega+k v_{z}\right) b_{\varphi}+\frac{d\left(V_{\mathrm{p}} b_{\varphi}\right)}{d r} \\
=r \frac{d \omega}{d r} b_{r}+\mathrm{Rm}^{-1}\left[\eta\left(\hat{D} b_{\varphi}+\frac{2 i m}{r^{2}} b_{r}\right)+\right. \\
\left.+\frac{d \eta}{d r}\left(\frac{d\left(r b_{\varphi}\right)}{r d r}-\frac{i m}{r} b_{r}\right)\right]
\end{gathered}
$$

where $V_{\mathrm{p}}=\eta d \ln \mu_{\mathrm{r}} / d r$, and

$$
\hat{D} \equiv \frac{1}{r} \frac{d}{d r}\left(r \frac{d}{d r}\right)-\frac{m^{2}+1}{r^{2}}-k^{2}
$$

is a Laplacian-type operator. Equations (7), (8) are written in non-dimensional form: distances are measured in units of the the radius $r_{0}$, velocity is measured in units of the longitudinal velocity on the axis of the flow, $U$. The magnetic diffusivity $\eta(r)$ is measured in units of $\eta_{\mathrm{fl}}$, which introduces the magnetic Reynolds number

$$
\mathrm{Rm} \equiv \mu_{0} \mu_{\mathrm{r}, \mathrm{fl}} \sigma_{\mathrm{fl}} r_{0} U=\frac{r_{0} U}{\eta_{\mathrm{fl}}}
$$

Correspondingly, in our non-dimensional units we have $\eta=1$ within the fluid and $\eta=\mu_{\mathrm{r}, \mathrm{f}} \sigma_{\mathrm{fl}} / \mu_{\mathrm{r}, \mathrm{sh}} \sigma_{\mathrm{sh}}$ in the surrounding shell.

In Eqs. (7) and (8), the longitudinal field component $b_{z}$ does not enter; once $b_{r}$ and $b_{\varphi}$ are known, it can be derived from the solenoidality condition $\nabla \cdot \mathbf{B}=0$, which yields

$$
b_{z}=\frac{i}{k r} \frac{d}{d r}\left(r b_{r}\right)-\frac{m}{k r} b_{\varphi}
$$

(note that $k \neq 0$ for all growing modes).

The shell $r_{0}<r<r_{1}$ can be treated as a special case of the above. In fact, in this case $\omega=v_{z}=0$, and the solution of (7), (8) is well-known and given by

$$
b_{r} \pm i b_{\varphi}=C_{ \pm} I_{m \pm 1}(\kappa r)+D_{ \pm} K_{m \pm 1}(\kappa r),
$$

where $\kappa=\sqrt{k^{2}+\mu_{0} \sigma \gamma}$, and $C_{ \pm}$and $D_{ \pm}$are complex constants determined by the boundary conditions. Here, $I_{m}(\cdot)$ and $K_{m}(\cdot)$ are modified Bessel functions, which are related to the Bessel and Hankel functions of the first kind by 16 .

$$
I_{m}(z)=i^{-m} J_{m}(i z), \quad K_{m}(z)=\frac{\pi}{2} i^{m+1} H_{m}^{(1)}(i z) .
$$

A straight-forward approach would be to use this analytical solution and match it to the solution in the inner 
region $r<r_{0}$ (requiring continuity of the tangential components of the electric field) and to a potential field in the exterior (see below). However, we decided to use a simpler approach and solve Eqs (7), (8) in the full region $0<r<r_{1}$ for the step-like profile

$$
\eta(r)=\left\{\begin{array}{ll}
1 & , r<r_{0} \\
\sigma_{\mathrm{fl}} / \sigma_{\mathrm{sh}} & , r_{0}<r<r_{1}
\end{array} .\right.
$$

Here, and for the one-dimensional results presented below, we have set $\mu_{\mathrm{r}}=1$ everywhere.

In the insulating outer domain $r>r_{1}$, the current density is zero, $\nabla \times \mathbf{B}=0$, and thus $\mathbf{B}$ can be expressed in terms of a scalar potential $P(r, \varphi, z, t)$,

$$
\mathbf{B}=-\nabla P .
$$

Solenoidality of $\mathbf{B}$ leads to the potential equation

$$
\frac{1}{r} \frac{\partial}{\partial r}\left(r \frac{\partial P}{\partial r}\right)+\frac{1}{r^{2}} \frac{\partial^{2} P}{\partial \varphi^{2}}+\frac{\partial^{2} P}{\partial z^{2}}=0,
$$

and due to the symmetry of the problem $P(r, \varphi, z, t)$ can be written in the form

$$
P(r, \varphi, z, t)=p(r) e^{\gamma t+i(m \varphi+k z)} .
$$

Equations (16) and (17) result in

$$
p^{\prime \prime}+\frac{1}{r} p^{\prime}-\left(\frac{m^{2}}{r^{2}}+k^{2}\right) p=0 .
$$

Solutions of (18) that are bounded for $r \rightarrow \infty$ have the form

$$
p(r)=C K_{m}(|k| r) .
$$

The boundary conditions at $r=r_{1}$ are obtained from the requirement of continuity of $\mathbf{b}$ on the outer border of the conducting shell. Together with Eqs. (6), (15), (17) and (19), this leads to

$$
\begin{aligned}
\frac{b_{r}\left(r_{1}\right)}{b_{\varphi}\left(r_{1}\right)} & =-i \frac{|k| r_{1}}{m} \frac{K_{m}^{\prime}\left(|k| r_{1}\right)}{K_{m}\left(|k| r_{1}\right)}, \\
\frac{b_{z}\left(r_{1}\right)}{b_{\varphi}\left(r_{1}\right)} & =\frac{k r_{1}}{m},
\end{aligned}
$$

where $K_{m}^{\prime}(x) \equiv d K_{m}(x) / d x$. Eliminating $b_{z}\left(r_{1}\right)$ from Eqs. (11) and (21) one finally gets

$$
b_{r}\left(r_{1}\right)+r_{1} b_{r}^{\prime}\left(r_{1}\right)=-i\left(\frac{k^{2} r_{1}^{2}}{m}+m\right) b_{\varphi}\left(r_{1}\right) \text {. }
$$

The inner boundary conditions follow from the regularity of $\mathbf{b}$ at $r=0$ and results in

$$
\begin{array}{llll}
b_{r}^{\prime}(0)=b_{\varphi}^{\prime}(0)=0, & \text { for } & & |m|=1, \\
b_{r}(0)=b_{\varphi}(0)=0, & \text { for } & & |m| \neq 1 .
\end{array}
$$

The system (7), (8), together with the boundary conditions (20), (22) and (23) is a non-selfadjoint eigenvalue problem. Dynamo action implies the existence of eigenvalues $\gamma$ with $\Re \gamma>0$. To obtain numerically the eigenvalues and eigenfunctions, we replace the derivatives by their finite-difference counterparts, using 200-800 grid points for this discretization. The resulting matrix eigenvalue problem is solved using the QR-algorithm.

\section{B. Results}

We have checked the one-dimensional numerical code by recalculating growth rates and critical magnetic Reynolds numbers from the literature. In particular, we have considered the case of (infinitely extended) insulating or perfectly conducting media surrounding the flow (for these tests we took $d=0.3 r_{0}$, and $\sigma_{\mathrm{sh}}=0.01 \sigma_{\mathrm{fl}}$ or $\left.\sigma_{\mathrm{sh}}=100 \sigma_{\mathrm{fl}}\right)$. Using the velocity profiles corresponding to the models by 6 ] or 7 ], we reproduced the corresponding critical magnetic Reynolds number with an accuracy of $5 \%$ or better. For the cases with analytical solutions 2, 3], the accuracy of our numerical results is better than $0.1 \%$.

In Ref. [9], it was demonstrated that the radial profile of the longitudinal velocity $v_{z}$ as measured in a water experiment is reasonably well approximated by

$$
v_{z}(r)=U \frac{\cosh (\xi)-\cosh \left(\xi r / r_{0}\right)}{\cosh (\xi)-1}
$$

for $\xi \approx 18$. Accordingly, we will use this parameterization throughout this paper, together with

$$
\omega(r)=\chi \frac{v_{z}(r)}{r_{0}}
$$

and treat $U, \xi$ and $\chi$ as free parameters. One advantage of the profile (24) is that it provides a smooth interpolation between the laminar Poiseuille solution (for $\xi \rightarrow 0$ ) and rigid-body motion (for $\xi \rightarrow \infty$ ). The latter limit corresponds to Ponomarenko's model; in practice, for $\xi=100, d=5 r_{0}$ and $\sigma_{\mathrm{sh}}=1$, the critical Reynolds number differs from Ponomarenko's solution by less than $0.1 \%$.

The threshold for dynamo action depends on the conductivity and the thickness of the shell and on the velocity profile. In Fig. 3 we show the dependency of the critical magnetic Reynolds number $\mathrm{Rm}_{*}$ on the shell thickness $d$ and the velocity profile parameter $\xi$ for a mode that is close to the easiest excitable one. In the first case (Fig. 31) the conductivities of fluid and shell are equal, in the second case (Fig. [3]) the shell conductivity is five times higher than that of the fluid, which approximately corresponds to the combination $\mathrm{Na}_{(1)} / \mathrm{Cu}$.

For the case $\sigma_{\mathrm{sh}} / \sigma_{\mathrm{fl}}=1$ shown in Fig. 37, the qualitative dependence of the dynamo threshold on the profile parameter $\xi$ is different for different values of the wall thickness. In the limit of a thin wall $(d \rightarrow 0), \mathrm{Rm}_{*}$ increases monotonously with $\xi$. This is explained by the decreasing capability of the shell to 'anchor' magnetic fields lines, which can partially be compensated by a wider shear region in the fluid, but becomes vital as the flow approaches rigid-body motion for large $\xi$. For a thicker shell, we find a very shallow minimum of $\operatorname{Rm}_{*}(\xi)$.

For the case of a highly conducting shell, $\sigma_{\mathrm{sh}}=5 \sigma_{\mathrm{f}}$, shown in Fig. 3] the situation is somewhat different. As $\xi$ increases and thus $\mathbf{v}$ approaches rigid motion, the threshold decreases, because now anchoring of the field 
a
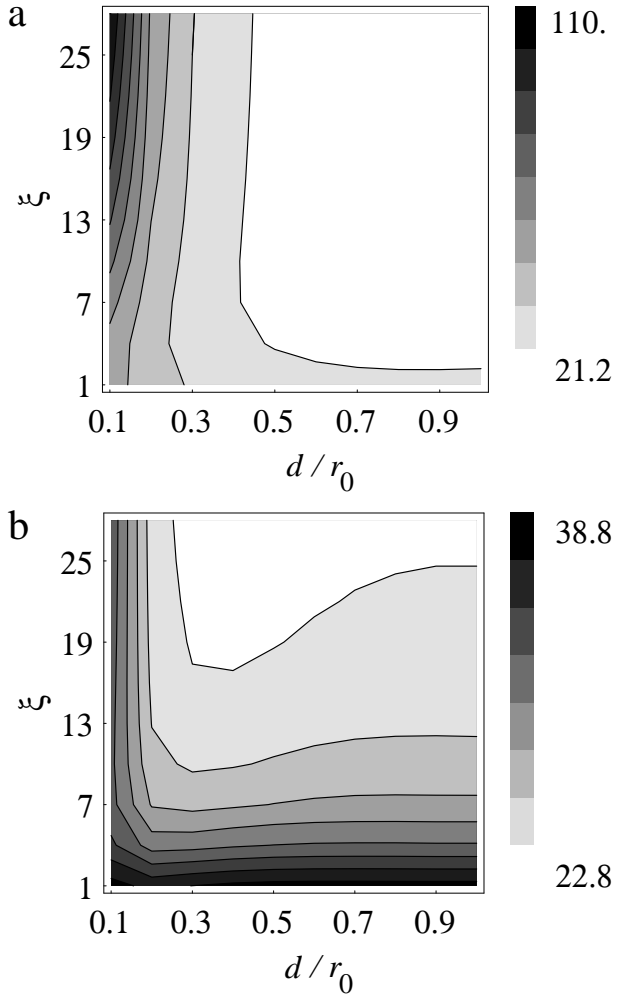

FIG. 3: Dependence of the critical magnetic Reynolds number $\mathrm{Rm}_{*}$ on the profile parameter $\xi$ and the shell thickness $d$. The graphs show isolines of $\operatorname{Rm}_{*}(d, \xi)$ for two conductivity ratios: a) $\sigma_{\mathrm{sh}}=\sigma_{\mathrm{fl}}$ and b) $\sigma_{\mathrm{sh}}=5 \sigma_{\mathrm{fl}}$. All data are for the mode $m=1, k=1 / r_{0}$, which is close to the fastest growing mode.

lines is always given (for the values $d \geq 0.1 r_{0}$ considered here), and the shearing of field lines is maximized by approaching the discontinuous velocity profile. For fixed $\xi>7$, the curve $\operatorname{Rm}_{*}(d)$ possesses a minimum at $d \approx 0.3-0.4$. Thus for the experimentally interesting values $\xi \approx 10-20$, there is an optimal thickness $d$ of the conducting shell.

Detailed investigation of the linear dynamo problem has shown that in an optimal experimental setup the electric conductivity of the shell should indeed be approximately five times larger than that of the liquid sodium. Provided the shell is thicker than about $15-20 \%$ of the inner torus radius $r_{0}$, the actual shell thickness has little effect on the excitation properties of the magnetic field. For mechanical reasons, the shell should be kept thin (about $10 \%$ of the inner torus radius), but for the numerical methods applied in this paper, it is more convenient to consider a slightly thicker shell (typically about $30 \%$ of the inner radius).

All these results were derived for $\sigma_{\text {ext }}=0$, i.e., an external insulator surrounding the shell, which corresponds well to the experimental setup. In the three-dimensional simulations presented in Sec. IV however, we can only approximate such an insulator by setting $\sigma_{\text {ext }}$ to a low but finite value. A discussion of the error involved by this approximation will be given in Sec. IVA

\section{THREE-DIMENSIONAL MODELS}

If the velocity field is time-dependent, Eqs. (2) and(3) can no longer be reduced to an eigenvalue problem, and we are faced with a Cauchy problem for the timeevolution of the magnetic field. Numerical solutions of this problem for the case of $z$-independent velocity fields have been presented in 9] and demonstrate that the screw dynamo should in principle work for the planned dynamo apparatus, which has an outer (torus) radius $R=0.4 \mathrm{~m}$ and an inner (pipe) radius $r_{0}=0.12 \mathrm{~m}$. To make quantitative predictions, however, the longitudinal dependence of the velocity field needs to be taken into account, since only after a time comparable to the braking time will all of the fluid be in helical motion and thus be able to generate magnetic field (see e.g. Fig. 7). In this Section, we present results for this time- and $z$-dependent flow, obtained with a three-dimensional MHD code. We still make the approximation of (periodic) cylindrical geometry and assume the velocity to be a given function of $r, z$ and $t$. The problem addressed now is thus a threedimensional kinematic dynamo problem with space- and time-dependent velocity.

The equation we are solving is the induction equation in the form

$$
\begin{aligned}
\frac{\partial \mathbf{A}}{\partial t} & =\mathbf{v} \times \mathbf{B}-\eta \mu_{0} \mu_{\mathrm{r}} \mathbf{j}+\eta_{0} \nabla \nabla \cdot \mathbf{A} \\
& =\left(\mathbf{v}+\eta \nabla \ln \mu_{\mathrm{r}}\right) \times \mathbf{B}+\eta \nabla^{2} \mathbf{A}-\left(\eta-\eta_{0}\right) \nabla \nabla \cdot \mathbf{A}(26)
\end{aligned}
$$

for the magnetic vector potential $\mathbf{A}$, from which the magnetic flux density $\mathbf{B}$ and the electric current density $\mathbf{j}$ are derived as $\mathbf{B}=\nabla \times \mathbf{A}$, and $\mathbf{j}=\nabla \times\left(\mathbf{B} / \mu_{0} \mu_{\mathrm{r}}\right)=$ $\left(\nabla \times \mathbf{B}-\nabla \ln \mu_{\mathrm{r}} \times \mathbf{B}\right) / \mu_{0} \mu_{\mathrm{r}}$. Equation (26) corresponds to the gauge in which the vector potential $\mathbf{A}$ and the scalar potential $\Phi$ are related by

$$
\eta_{0} \nabla \cdot \mathbf{A}+\Phi=0,
$$

where the constant parameter $\eta_{0}$ (introduced for purely numerical reasons) is arbitrary and was chosen equal to the magnetic diffusivity $\eta_{\mathrm{fl}}$ of the fluid.

As before, we use the parameterization (24), (25) for the radial profiles of $v_{z}$ and $\omega$, where $\chi$ is either 1 (in Sec. IVA or $z$-dependent and determined from a more sophisticated model (Sec. IVB). All dimensional results in this section refer to the fiducial experimental apparatus [9] with the parameters given in Table —

For numerical reasons we have smoothed the radial profile of magnetic diffusivity $\eta(r)$; the resulting profile is shown in Fig. 4 the ratio $\eta_{\mathrm{sh}} / \eta_{\mathrm{fl}}$ is equal to 0.2 . We embed the cylinder in a region of enhanced magnetic diffusivity; while an insulating medium corresponds to $\eta=\infty$ and very large values of magnetic diffusivity are thus desirable, numerical requirements limit the values of $\eta$ strongly. We found that $\eta_{\text {ext }}=5 \eta_{\mathrm{fl}}$ still results in tolerable time steps, while providing already a 
TABLE I: Parameters and results for the different numerical calculations presented in Sec. IV] Parameters common to all models are: torus radius $R=0.4 \mathrm{~m}$ (resulting in a cylinder length $L_{z} \approx 2.5 \mathrm{~m}$ ), pipe radius $r_{0}=0.12 \mathrm{~m}$, outer shell radius $r_{1}=0.16 \mathrm{~m}$, initial angular velocity of the torus (before braking) $\Omega_{0}=310 \mathrm{~s}^{-1}$, and the magnetic diffusivities $\eta_{\mathrm{sh}}=0.016 \mathrm{~m}^{2} / \mathrm{s}, \eta_{\mathrm{ext}}=0.4 \mathrm{~m}^{2} / \mathrm{s} . \quad T_{b}$ denotes the braking time. The amplification factors $\Gamma_{\text {net }}$ and $\Gamma_{\max }$ are defined in Eqs. (29).

\begin{tabular}{lcccll}
\hline \hline Label & $\eta_{\mathrm{fl}}\left(\mathrm{m}^{2} / \mathrm{s}\right)$ & initial field & $T_{\mathrm{b}}(\mathrm{s})$ & $\Gamma_{\text {net }}$ & $\Gamma_{\max }$ \\
\hline Run 1 & 0.08 & random & 0.1 & 87 & $4.4 \times 10^{3}$ \\
Run 1b & 0.08 & $k=k_{3}$ & 0.1 & $1.2 \times 10^{3}$ & $2.1 \times 10^{4}$ \\
Run 2 & 0.04 & random & 0.1 & $6.8 \times 10^{4}$ & $1.0 \times 10^{6}$ \\
Run 3 & 0.08 & random & 0.2 & $<1$ & $1.9 \times 10^{2}$ \\
Run 4 & 0.04 & random & 0.2 & 860 & $3.2 \times 10^{4}$ \\
\hline \hline
\end{tabular}

good approximation to the case of a surrounding insulator (cf. Sec. IVA).

We use a numerical scheme that is of 6th order in space, and perform 3rd-order explicit time-stepping. Despite the cylindrical geometry, we use a Cartesian grid, which avoids the special treatment the axis would otherwise require. The same approach was used with a similar code in 13] to model nonlinear screw-dynamo action in spiral Couette flow. Our boundary conditions are periodic in the vertical direction (corresponding to the model employed in Sec. III). In the horizontal direction, on the Cartesian boundaries of the high-diffusivity region, we require the magnetic field to be normal to the boundaries,

$$
A_{\perp}=0, \quad \frac{\partial}{\partial n} \mathbf{A}_{\|}=\mathbf{0},
$$

where $\perp$ and $\|$ indicate the directions normal and parallel to the boundary, and $\partial / \partial n$ denotes the normal derivative. Conditions (28) imply the absence of currents across the boundaries which makes it a qualitative local approximation to the case of a surrounding insulator.

We start with a smoothed random magnetic field. By this we mean that the vector potential is set to a zerocorrelated random field in all grid points within $r<r_{0}$ and to zero otherwise; we then let the magnetic field diffuse for a fraction $\approx 0.2$ of the diffusion time $r_{0}^{2} / \eta_{\mathrm{f}}$, which reduces the amplitude of the high-wavenumber modes (which decay quickly anyway) and causes the field to slightly extend into the shell and, very weakly, into the external medium.

For the following discussion, it is helpful to characterize the modes by their longitudinal wave number $k$. Strictly speaking, this is only appropriate for $z$-independent velocity fields like the tests given in Sec.IVA or towards the end of the dynamical calculations. In these cases, individual magnetic modes will have wave numbers $k_{n}=n k_{1}$ which are multiples of $k_{1} \equiv 2 \pi / L_{z}$. However, in many cases one can clearly count the number $n$ of reversals of

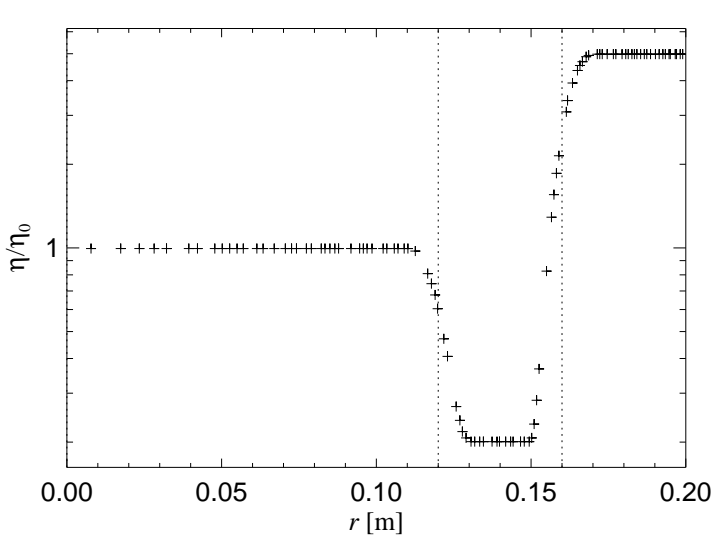

FIG. 4: Radial profile of magnetic diffusivity as used in the numerical calculations for the resolution $\delta x=0.011 \mathrm{~m}$. The region $0 \leq r<r_{0}$ corresponds to the fluid; $r_{0}<r<r_{1}$ defines the shell, and $r>r_{1}$ represents a poorly conducting medium surrounding the shell. Note that for the tests at $\delta x=0.0056 \mathrm{~m}$, the profile was steeper and more step-function like.

$B_{z}$ along the cylinder, and we will identify this number $n$ with a corresponding wave number $k_{n}$. The azimuthal wave number of any relevant mode is $m= \pm 1$ (except for Sec. IVC); for clarity, we will refer only to $m=+1$.

\section{A. Steady velocities}

To test the code and get an estimate of the error introduced by the approximations discussed above (namely the approximation of the surrounding insulator by a region of enhanced magnetic diffusivity, and the smoothed radial $\eta$-profile as shown in Fig. (4), we have compared results of the three-dimensional code with those from the eigenvalue problem in Sec. III for steady flows.

Figure [5 shows the growth rate $\Re \gamma$ of the magnetic field obtained by the three-dimensional code (diamonds and asterisks) as a function of $\mathrm{Rm}$. The data points are in good agreement with the reference curve, which was obtained with the one-dimensional code and has an estimated error of $\leq 1 \%$.

The validity of approximating the insulating exterior region by a low-conductivity medium can be explicitly assessed from Fig. [6] where the critical magnetic Reynolds number $\mathrm{Rm}_{*}$ is shown as a function of $\sigma_{\mathrm{sh}} / \sigma_{\mathrm{ext}}$. An insulator is represented by the limit $\sigma_{\mathrm{sh}} / \sigma_{\text {ext }} \rightarrow \infty$. It is evident that $\sigma_{\mathrm{sh}} / \sigma_{\text {ext }}=25$ results in a generation threshold quite close to that for $\sigma_{\text {ext }} \rightarrow 0$. This holds particularly for $\sigma_{\mathrm{sh}}=5 \sigma_{\mathrm{fl}}$, and is intuitive because the magnetic field is located around the interface between the liquid and the shell, with low amplitude at the outer shell surface. 


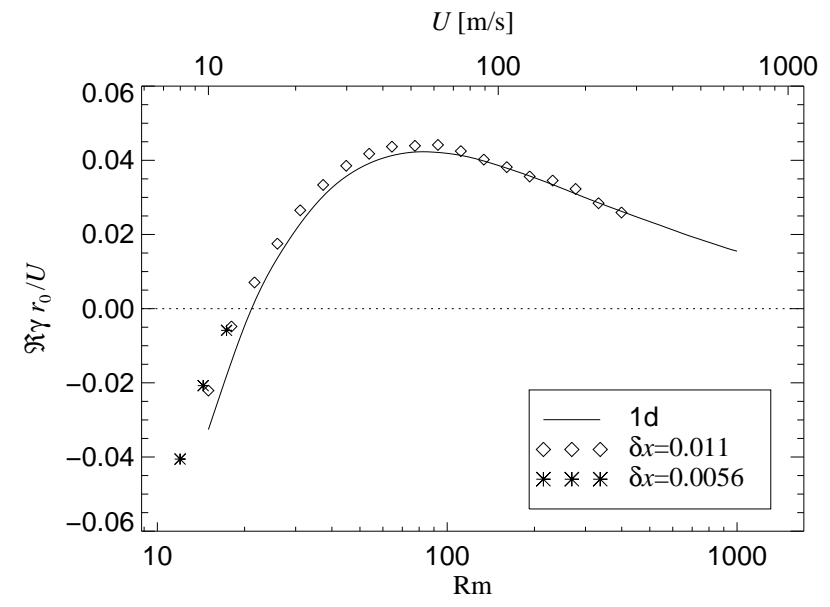

FIG. 5: Kinematic growth rate $\Re \gamma$ as a function of magnetic Reynolds number for $d / r_{0}=0.333$. Solid line: onedimensional model with 200 radial points. Diamonds $(\diamond)$ and asterisks $(*)$ : values obtained with the three-dimensional code at resolution $\delta x=\delta y=0.011$ and $\delta x=\delta y=0.0056$, respectively. The longitudinal wave number is $k=k_{3}$ in all cases, i.e., the longitudinal extent of the pipe is three wavelengths of the magnetic mode. The second abscissa shows the velocity in an apparatus characterized by $r_{0}=0.12 \mathrm{~m}, \eta=0.08 \mathrm{~m}^{2} / \mathrm{s}$.

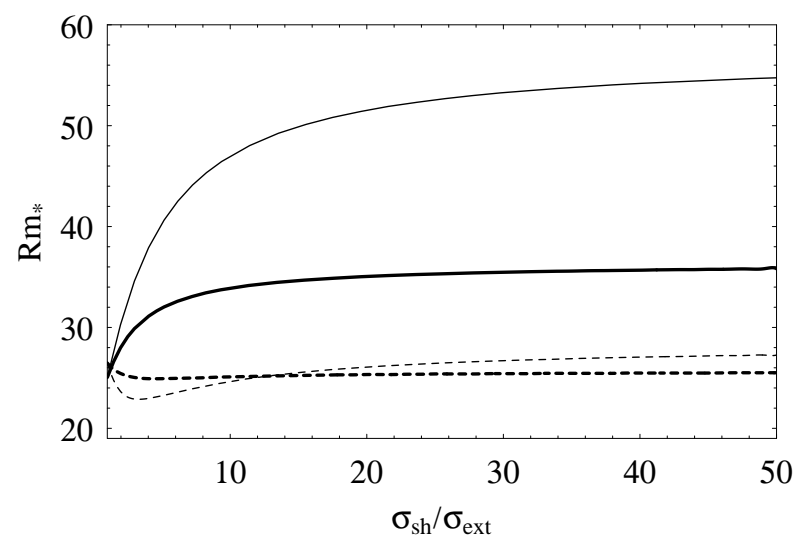

FIG. 6: Generation threshold $\mathrm{Rm}_{*}$ as a function of the conductivity ratio $\sigma_{\mathrm{sh}} / \sigma_{\mathrm{ext}}$ as obtained with the one-dimensional model described in Sec. III The solid line is for $\sigma_{\mathrm{sh}}=\sigma_{\mathrm{ff}}$, the dashed line for $\sigma_{\mathrm{sh}}=5 \sigma_{\mathrm{fl}}$. Thin lines correspond to a thin shell, $d=0.15 r_{0}$, thick lines to a thicker shell, $d=0.3 r_{0}$. All values are for $\xi=10$.

\section{B. Dynamo action in a time-dependent helical flow}

Having confirmed the accuracy of our numerical code for the case of constant velocities, we are now in the position to investigate the problem for a time- and $z$ dependent velocity field. We consider only the case of one single diverter in the channel, because it turns out that additional diverters, while shortening the transition time after which the flow is fully helical, have a negative impact on the maximum flow velocity and accelerate the decay of the velocity $U(t)$. The net effect of increasing
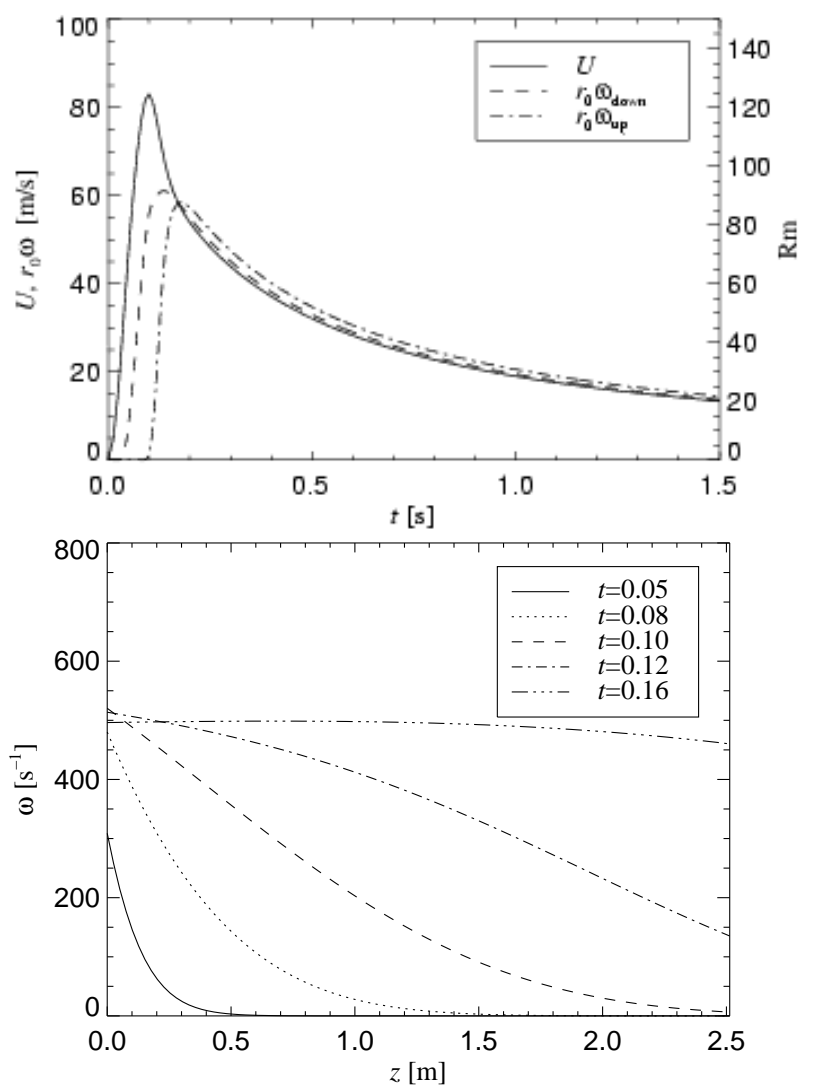

FIG. 7: Evolution of the velocity structure with time. The braking time is $T_{\mathrm{b}}=0.1$, and only one diverter is used. Top: velocity as a function of time. The three curves represent the longitudinal velocity on the axis, $U=v_{z}(r=0)$ (solid line), and the angular velocity on the axis (multiplied by $r_{0}$ ), $r_{0} \omega(r=0)$, downstream and upstream of the diverter (dashed and dashed-dotted line, respectively). Bottom: Angular velocity $\omega(r=0)$ along the cylinder for different times.

the number of diverters has always been found to be unfavorable for the dynamo.

We take the longitudinal velocity $v_{z}(r, t)$ and angular velocity $\omega(r, z, t)$ from the hydrodynamical model described in Ref. [9], together with the radial dependence (24), (25). At the diverter position, we slightly smooth the velocity field to avoid discontinuities. We first consider a short braking time of $T_{\mathrm{b}}=0.1 \mathrm{~s}$. Figure 7 shows the time dependence of $v_{z}$ and $\omega$ on the axis. The maximum angular velocity is reached only after the end of the braking phase and the rotating zone needs an additional $\approx 0.05 \mathrm{~s}$ to fill the whole torus.

Figure 8 shows the time-dependence of the root-meansquare magnetic field for Run 1, compared to the 'optimistic' and 'pessimistic' extrapolations from longitudinally uniform models: the 'pessimistic' model is obtained by adopting the angular velocity upstream of the diverter for any $z$, while the 'optimistic' model corresponds to using the downstream value [9]. For $0<t<T_{\mathrm{b}}=0.1 \mathrm{~s}$, the initial field decays towards a simpler structure (larger scales). During the time interval $0.1 \mathrm{~s}<t<0.2 \mathrm{~s}$, the re- 


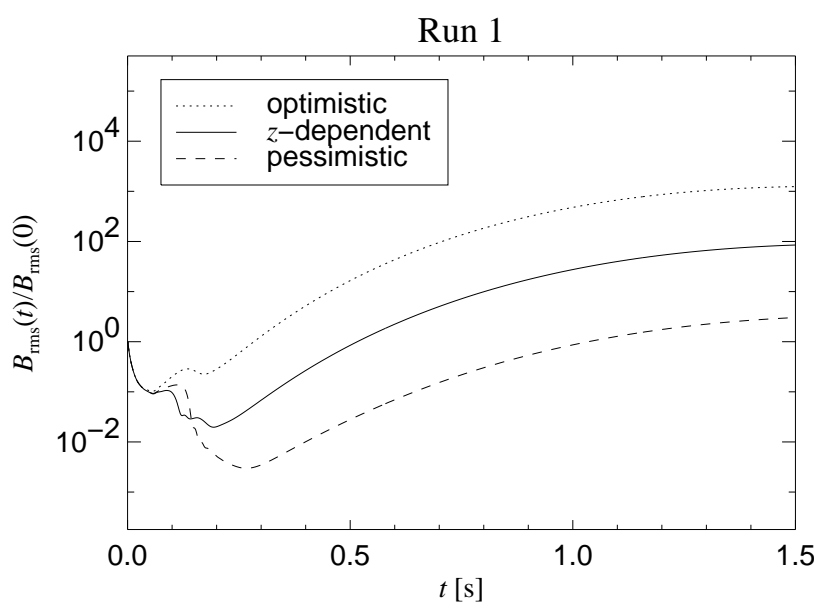

FIG. 8: Root-mean-square magnetic flux density as a function of time for the three-dimensional model Run 1. For comparison with the full model (labeled ' $z$-dependent'), the results of two $z$-independent simulations ('optimistic' and 'pessimistic' are also shown. The spatial resolution here and for all following graphs is $\delta x=\delta y=0.011, \delta z=0.084$.

sulting mode is restructured into $m=1, k=k_{3}$, which is eventually the fastest growing mode. We can define the net and maximum amplification factors as

$$
\Gamma_{\text {net }} \equiv \frac{\max _{t} B_{\mathrm{rms}}(t)}{B_{\mathrm{rms}}(0)}, \quad \Gamma_{\mathrm{max}} \equiv \frac{\max _{t} B_{\mathrm{rms}}(t)}{\min _{t} B_{\mathrm{rms}}(t)} .
$$

The corresponding values for Run 1 are $\Gamma_{\text {net }}=87$, $\Gamma_{\max }=4.4 \times 10^{3}$ (see Table \).

The geometrical evolution of the magnetic field structure is illustrated by Fig. 9] where isosurfaces of the magnetic flux density $|\mathbf{B}|$ are shown at eight different times. Note how the initial mode $m=1, k=0$ (the slowest decaying mode with vanishing vertical net magnetic flux $\Phi_{\mathrm{m}} \equiv \int B_{z} d x d y$ in a non-helical flow) is transformed into $k=k_{1}$ and eventually $k=k_{3}$. Given that modes with different vertical wave numbers $k$ evolve approximately independent from each other (since the flow always has some $z$-dependence, there is some mixing between the modes, however), the field $k=k_{1}$ which is dominant at $t=0.15$ does not provide a good seed field for the later growth of the final mode $k=k_{3}$.

This highlights the importance of the initial field configuration for the net growth of the magnetic field strength during the experiment. To further investigate the situation, we show in Fig. 10 the growth curve from a different simulation Run 1b, with identical parameters, but using as initial field the final field (at $t=1.5 \mathrm{~s}$ ) from Run 1. During the first $0.15 \mathrm{~s}$, the field decays, since the flow is not helical everywhere. After $t=0.15 \mathrm{~s}$, the mode $k=k_{3}$ grows by a factor of $2.1 \times 10^{4}$ (maximum growth), and the net growth is $1.2 \times 10^{3}$. Thus, the choice of the initial field can have a dramatic effect on the amplification factors.

\section{Enhanced magnetic permeability}

For a paramagnetic or ferromagnetic fluid, the magnetic diffusivity $\eta_{\mathrm{fl}}$ is

$$
\eta_{\mathrm{fl}}=\frac{1}{\mu_{0} \mu_{\mathrm{r}, \mathrm{f}} \sigma_{\mathrm{fl}}},
$$

which shows that one possible option of increasing the magnetic Reynolds number is to increase the relative magnetic permeability $\mu_{\mathrm{r}}$. In an experimental setup like that of the Perm experiment, where there are no movable parts within the fluid, such an enhancement of $\mu_{\mathrm{r}}$ can be safely achieved by adding ferromagnetic particles to the fluid. Direct measurements of the effective permeability of two-phase liquids indicate that $\mu_{\mathrm{r}}$ can be at most $\approx 2$ if reasonable flow properties are to be maintained [17].

To assess the consequences of such an increase in Rm, we have carried out as Run2 a simulation with $\mu_{\mathrm{r}, \mathrm{f}}=2$, which implies a two times lower value of $\eta_{\mathrm{fl}}$ (while $\eta_{\mathrm{sh}}$ and $\eta_{\text {ext }}$ are fixed to their previous values). The profile $\mu_{\mathrm{r}}(r)$ was a smoothed step profile analogous to the one

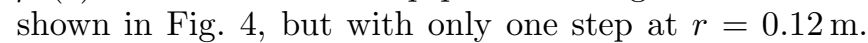
Figure 10p shows that the net and maximum amplification factors are now increased by about three orders of magnitude. The main effect is not a faster growth of $B$, but rather a reduced decay of the initial field, followed by a much prolonged growth phase. These observations can be understood from Fig. 5 as follows. Since the dependency $\Re \gamma(\mathrm{Rm})$ is non-monotonous, doubling the magnetic Reynolds number will not necessarily increase the growth rate, but in fact reduce it if $\mathrm{Rm} \gtrsim 40$. On the other hand, the flow will remain supercritical $(\mathrm{Rm}>21)$ for a much longer time span.

The fourth line in Fig. 10] shows an artificial $z$ dependent run, where the 'paramagnetic pumping' velocity $\mathbf{V}_{\mathrm{p}}$ was set to zero; this would correspond to the case where $\eta_{\mathrm{ff}}$ is reduced by enhancing $\sigma_{\mathrm{ff}}$, rather than $\mu_{\mathrm{r}, \mathrm{fl}}$. The comparison shows that the pumping term is indeed important for the field growth, since without it the net growth would be reduced to about 3400 .

Another interesting finding is that the rms magnetic field for the full model is no longer contained in the interval spanned by the 'optimistic' and the 'pessimistic' variants. Rather, the rms filed for the 'pessimistic' run overtakes the $z$-dependent one and closely approaches even the 'optimistic' run. This unexpected behavior is connected to the presence of several growing modes and can be understood by a closer look at the modal structure of the solutions. In Fig. [1] we show the time evolution of individual modes identified by their vertical wave number $k$. Strictly speaking, only for $z$-independent velocity profiles (i.e. for the 'optimistic' and 'pessimistic' models) each dynamo mode will be characterized by a unique value of $k$ (together with the azimuthal wave number $m$ and a radial one), but the spectral analysis (for the $z$ direction) is a very helpful tool even if this condition is not satisfied. 

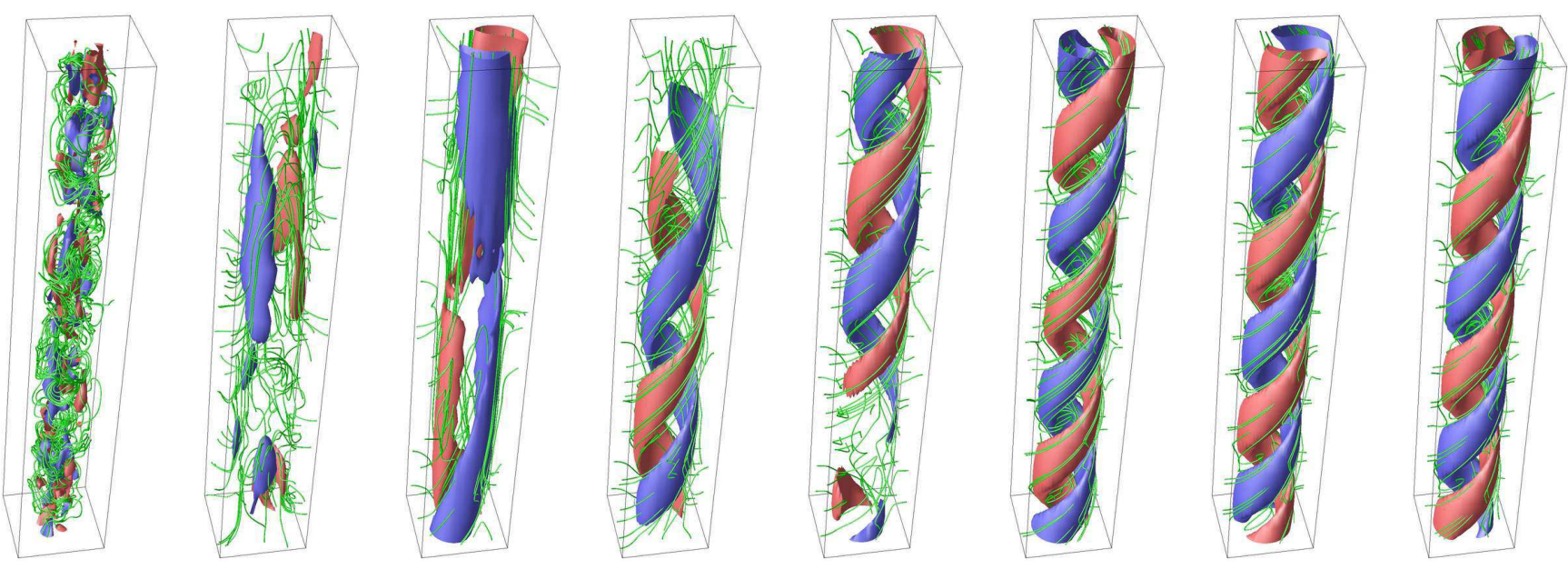

FIG. 9: Structure of the magnetic field for different times of Run 1. From left to right, the times are 0.0, 0.05, 0.1, 0.15, 0.2, $0.3,0.4$ and $0.5 \mathrm{~s}$; the braking time is $T_{\mathrm{b}}=0.1 \mathrm{~s}$. The surfaces are isosurfaces of the magnetic field strength (red: $B_{z}>0$, blue: $\left.B_{z}<0\right)$. The lines are magnetic field lines. The diverter is located at the bottom; the direction of the flow is upwards.
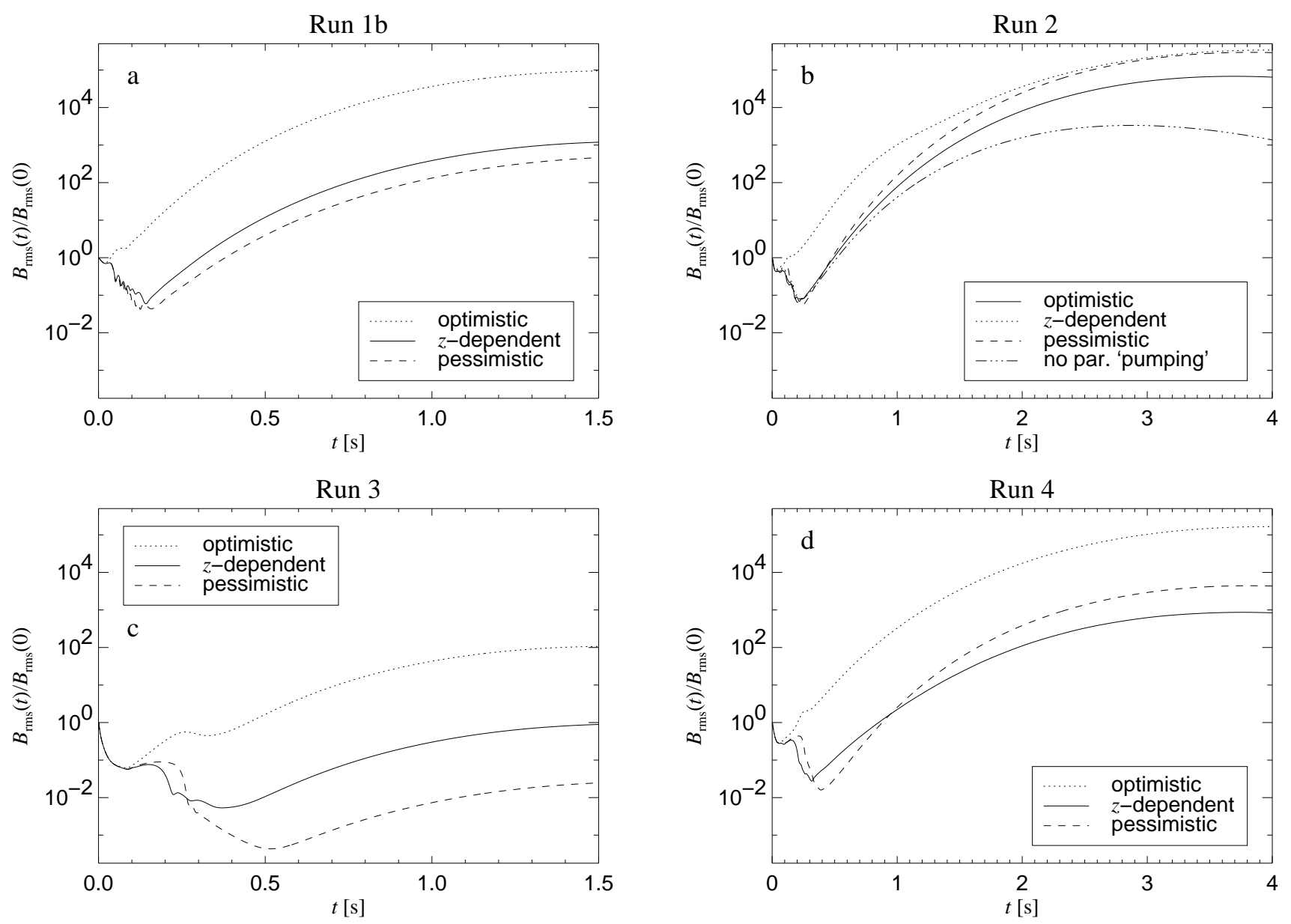

FIG. 10: Like Fig. 8 but for different parameters. a) Like Run 1, but starting with a clean $k=k_{3}$ mode (Run 1b). b) Like Run 1, but with a two times lower magnetic diffusivity (achieved by enhancing the magnetic permeability to $\mu_{\text {rel }}=2$; Run 2 ); note the different time interval plotted and accordingly the much longer growth phase compared to Run 1; the fourth line ( - ...) shows the results for a model where the 'paramagnetic pumping' velocity $\mathbf{V}_{\mathrm{p}}$ was artificially set to zero. c) Like Run 1 , but with a longer braking time $T_{\mathrm{b}}=0.2$ (Run 3). d) Like Run 2, but with a longer braking time $T_{\mathrm{b}}=0.2($ Run 4 ). 

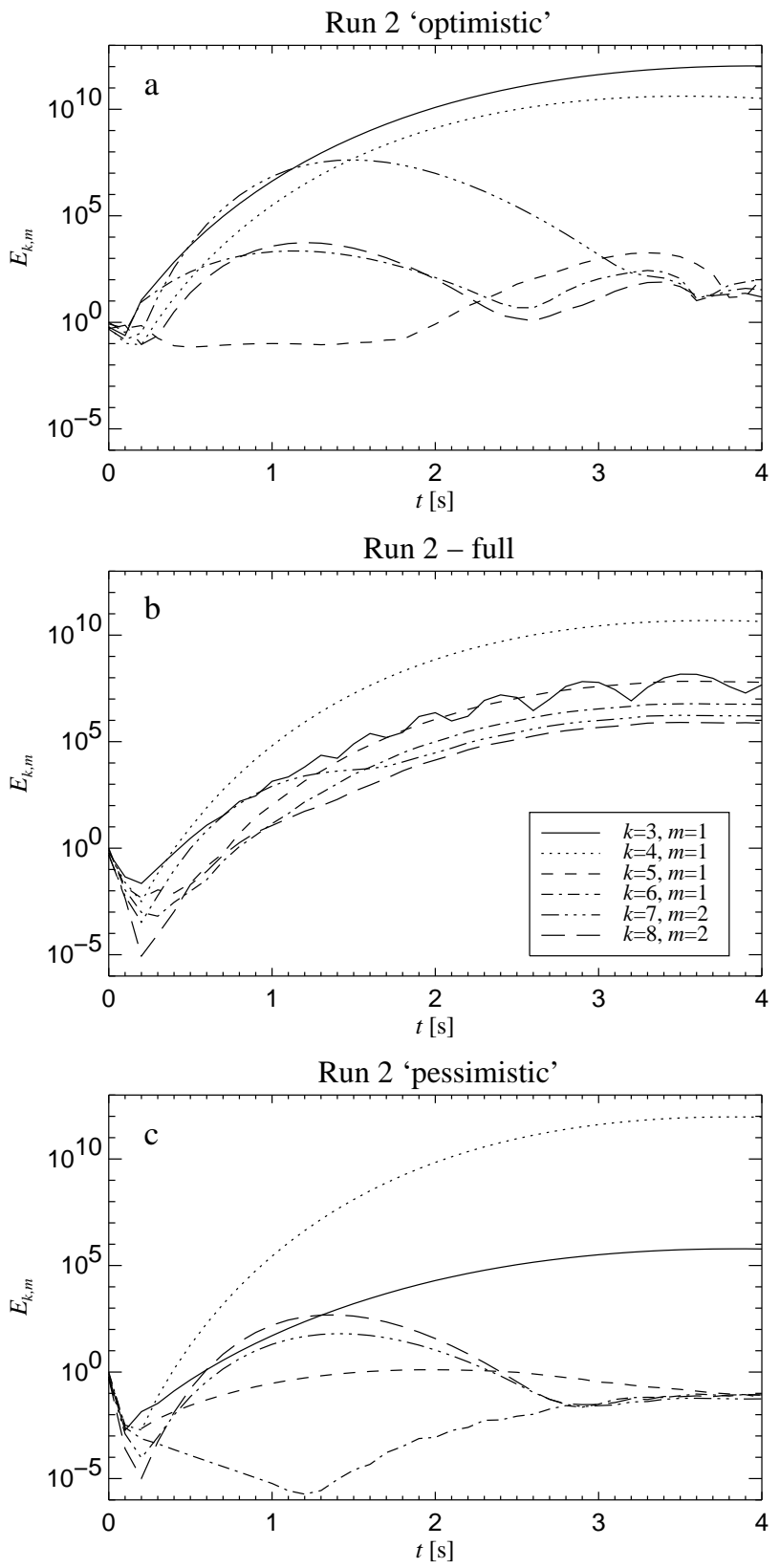

FIG. 11: Evolution of Fourier modes for Run2. a) 'optimistic' model; b) full model; c) 'pessimistic' model. Shown is the energy $E_{k, m}$ of the modes normalized such that the maximum at $t=0$ is 1 . The oscillations of the mode $k=k_{3}$ in the second plot are connected to a change in the radial structure, i.e. most probably due to different radial modes.

As can be seen from Fig. 11] in the 'optimistic' model the mode $k=k_{3}$ (and $m=1$ ) dominates, while for the full and 'pessimistic' models $k=k_{4}$ (and $m=1$ ) is the dominating mode. For most of the time, the growth rate of the modes $k_{3}$ and $k_{4}$ are comparable, which indicates that a wave number between $k_{3}$ and $k_{4}$ would be optimal, but is excluded by the geometrical setup. Other growing modes include $k=k_{7}, m=2$, which was never encoun- tered for Run 1, where the magnetic Reynolds number was two times lower.

Since the magnetic diffusion time $r_{1}^{2} / \eta=0.36 \mathrm{~s}$ is at least comparable to the evolution time of the flow, the growth of the modes is never just determined by the growth rate for the current value of $\mathrm{Rm}$, but rather involves the history of the given mode. For the full, $z$ dependent model, an additional important factor is the action of the diverter, which for all times (but particularly during the first $0.2 \mathrm{~s}$ ) introduces a $z$-dependence of the velocity field and thus mixes energy from the dominating mode $k=k_{4}$ into other modes. This is the reason why in Fig. 11] the different modes show quite similar behavior after $t \approx 0.3 \mathrm{~s}$. Although the same effect will occur for $\mu_{\mathrm{r}}=1$, too, the resulting energy loss from the dominating mode will be weaker there, because the shorter magnetic diffusion time allows the leading mode to better adjust to the $z$-dependence of the velocity field.

As a result of these effects, drawing conclusions for the full problem from the simple 'pessimistic' and 'optimistic' models can be quite problematic.

\section{Slower braking of the torus}

To assess the effect of a longer braking phase, we have carried out calculations with $T_{\mathrm{b}}=0.2 \mathrm{~s}$; the results are shown in Fig. 10r for $\mu_{\mathrm{r}}=1$ (Run 3) and in Fig. 10 for $\mu_{\mathrm{r}}=2$ (Run 4). In the case of liquid sodium without admixed ferromagnetic particles, the maximum growth is diminished to about 100 and the net growth is less than 1, i.e., at the end of the experiment the magnetic energy is lower than it was for the seed field. Only with the enhanced value $\mu_{\mathrm{r}}=2$ we obtain net growth, which is now larger than in Run 1, but still significantly lower than in Run 2.

\section{CONCLUSION}

The results presented here confirm the conclusion that the planned Perm dynamo experiment is realistic and will be able to yield field amplification factors of about $10^{3}$ or more. A thin, highly conducting shell is crucial for the dynamo process and its role is well understood.

Short braking times are necessary for the dynamo, and a time of $T_{\mathrm{b}}=0.1 \mathrm{~s}$ as is intended for the Perm experiment [9] will be sufficient.

Enhancing the magnetic permeability by adding ferromagnetic particles to the liquid sodium would further enhance the amplification factor, but this is not crucial for the success of the experiment. For longer braking time $T_{\mathrm{b}}=0.2 \mathrm{~s}$, however, enhancing $\mu_{\mathrm{r}}$ is required to obtain net growth of the field at all. The enhanced magnetic Reynolds numbers for $\mu_{\mathrm{r}}=2$ cause a number of modes to grow (which complicates the analysis), and aggravates the loss of energy from the leading mode due to the diverter. 
The final amplification factor strongly depends on the initial magnetic field configuration, and choosing a suitable seed field can be vital for obtaining good results. For example, relying on the terrestrial background field may be a bad choice, as a uniform field penetrating a torus has only components $m=0, k= \pm k_{1}$ and $m=1$, $k=0$ while a good seed field should have a significant amount of energy in the optimal mode $m=1, k=k_{3}$. A sophisticated arrangement of small permanent magnets may be able to provide such a field, but one should even consider an arrangement of coils that allows for a net current through the inner part of the torus. This question certainly requires more detailed investigations.

\section{Acknowledgments}

We acknowledge partial financial support from ISTC under grant 2021, from the Leverhulme Trust (Grant F/125/AL) and from the Deutsche Forschungsgemeinschaft (Grant No. STI 65/12-1). The three-dimensional numerical calculations have been carried out on the PPARC supported parallel computers in St. Andrews and Leicester.
[1] D. Lortz, Plasma Phys. 10, 967 (1968).

[2] Y. B. Ponomarenko, J. Appl. Mech. Tech. Phys. 14, 775 (1973), translation from Zh. Prikl. Mekhaniki i Tekhn. Fiziki.

[3] A. Gailitis and Y. Freiberg, Magnetohydrodynamics 12, 127 (1976).

[4] A. Ruzmaikin, D. Sokoloff, and A. Shukurov, J. Fluid Mech. 197, 39 (1988).

[5] A. D. Gilbert, Geophys. Astrophys. Fluid Dyn. 44, 241 (1988).

[6] A. A. Solovyov, Dokl. Akad. Nauk SSSR 282, 44 (1985).

[7] E. A. Lupyan and A. Shukurov, Magnetohydrodynamics 28, 234 (1992).

[8] J. Léorat, Magnetohydrodynamics 31, 367 (1995).

[9] P. Frick, V. Noskov, S. Denisov, S. Khripchenko, D. Sokoloff, R. Stepanov, and A. Sukhanovsky, Magnetohydrodynamics 38, 143 (2002).

[10] A. M. Soward, Geophys. Astrophys. Fluid Dyn. 53, 81
(1990).

[11] A. D. Gilbert and Y. Ponty, Geophys. Astrophys. Fluid Dyn. 93, 55 (2000).

[12] A. P. Bassom and A. D. Gilbert, J. Fluid Mech. 343, 375 (1997).

[13] W. Dobler, A. Shukurov, and A. Brandenburg, Phys. Rev. E 65, 036311 (2002), astro-ph/0105484.

[14] S. A. Denisov, V. I. Noskov, D. D. Sokolov, P. G. Frick, and S. Y. Khripchenko, Dokl. Akad. Nauk 365, 478 (1999).

[15] K.-H. Rädler and R. Stepanov, in The Vth International Conference PAMIR (2002), pp. VI-77.

[16] M. Abramowitz and I. A. Stegun, Pocketbook of Mathematical Functions (Harri Deutsch, Thun, Frankfurt, 1984).

[17] P. Frick, S. Khripchenko, S. Denisov, D. Sokoloff, and J.-F. Pinton, Europ. Phys. J. B 25, 399 (2002). 